\title{
The Hummingbird-2 Lightweight Authenticated Encryption Algorithm
}

\author{
Daniel Engels, Markku-Juhani O. Saarinen, \\ Peter Schweitzer, and Eric M. Smith \\ REVERE SECURITY \\ 4500 Westgrove Drive, Suite 335, Addison, TX 75001, USA. \\ \{daniel.engels,mjos, pater.schweitzer, eric.smith\} \\ areveresecurity.com
}

\begin{abstract}
Hummingbird-2 is an encryption algorithm with a 128-bit secret key and a 64-bit initialization vector. Hummingbird-2 optionally produces an authentication tag for each message processed. Like it's predecessor Hummingbird-1, Hummingbird-2 has been targeted for low-end microcontrollers and for hardware implementation in lightweight devices such as RFID tags and wireless sensors. Compared to the previous version of the cipher, and in response to extensive analysis, the internal state has been increased to 128 bits and a flow of entropy from the state to the mixing function has been improved. In this paper we present the Hummingbird-2 algorithm, its design and security arguments, performance analysis on both software and hardware platforms, and timing analysis in relation to the ISO 18000-6C protocol.
\end{abstract}

Keywords: Hummingbird cipher, constrained devices, lightweight cryptography, ISO 18000-6C.

\section{Introduction}

Authenticated encryption algorithms provide confidentiality and integrity protection for messages using a single processing step. This results in performance and cost advantages, especially when the algorithm is implemented in hardware.

Hummingbird- 2 is an authenticating encryption primitive that has been designed particularly for resource-constrained devices such as RFID tags, wireless sensors, smart meters and industrial controllers. Hummingbird-2 can be implemented with very small hardware or software footprint and is therefore suitable for providing security in low-cost ubiquitous devices.

The design described in this paper is an evolutionary step from Hummingbird$1[8,10,11]$ and was developed in part as a response to the cryptanalysis of the cipher presented in [20]. Hummingbird-2 is resistant to all previously known cryptanalytic attacks. 
Table 1. S-Boxes used in Hummingbird-2.

\begin{tabular}{|c|cccccccccccccccc|}
\hline$x$ & 0 & 1 & 2 & 3 & 4 & 5 & 6 & 7 & 8 & 9 & 10 & 11 & 12 & 13 & 14 & 15 \\
\hline$S_{1}(x)$ & 7 & 12 & 14 & 9 & 2 & 1 & 5 & 15 & 11 & 6 & 13 & 0 & 4 & 8 & 10 & 3 \\
$S_{2}(x)$ & 4 & 10 & 1 & 6 & 8 & 15 & 7 & 12 & 3 & 0 & 14 & 13 & 5 & 9 & 11 & 2 \\
$S_{3}(x)$ & 2 & 15 & 12 & 1 & 5 & 6 & 10 & 13 & 14 & 8 & 3 & 4 & 0 & 11 & 9 & 7 \\
$S_{4}(x)$ & 15 & 4 & 5 & 8 & 9 & 7 & 2 & 1 & 10 & 3 & 0 & 14 & 6 & 12 & 13 & 11 \\
\hline
\end{tabular}

The Hummingbird-2 does not directly fall to either traditional stream cipher or block cipher categories as it inherits properties from both. In this sense Hummingbird-2 resembles the Helix and Phelix proposals [9, 16, 22]. Since Hummingbird-2 operates on 16-bit blocks, more efficiency can be realized in applications that chirp small messages, such as RFID devices or wireless sensors. This also makes it easy to layer in security in various protocol schemes.

This paper is structured as follows: A formal description of Hummingbird-2 is contained in Section 2. Section 3 has the preliminary results of cryptanalysis of the cipher. Software and hardware implementations are described in Section 4, together with ISO 18000-6C timing information and comparison in Section 5. Conclusions can be found in Section 6. Appendix A contains a set of implementation test vectors for Hummingbird-2.

\section{Description of Hummingbird-2}

The Hummingbird-2 cipher has a 128-bit secret key $K$ and a 128-bit internal state $R$ which is initialized using a 64-bit Initialization Vector $I V$. These variables are accessed as vectors of 16-bit words:

$$
\begin{aligned}
K & =\left(K_{1}, K_{2}, K_{3}, K_{4}, K_{5}, K_{6}, K_{7}, K_{8}\right), \\
R & =\left(R_{1}, R_{2}, R_{3}, R_{4}, R_{5}, R_{6}, R_{7}, R_{8}\right), \\
I V & =\left(I V_{1}, I V_{2}, I V_{3}, I V_{4}\right) .
\end{aligned}
$$

Hummingbird-2 is entirely built from operations on 16-bit words: the exclusiveor operation on words $(\oplus)$, addition modulo $65536(\boxplus)$ and a nonlinear mixing function $f(x)$.

\subsection{Nonlinear functions $f(x)$ and WD16(x, $a, b, c, d)$}

The nonlinear mixing function $f$ consists of four-bit S-Box permutation lookups on each nibble of the word, followed by a linear mix. 
The Hummingbird-2 S-Boxes $S_{1}, S_{2}, S_{3}$ and $S_{4}$ are given Table $1 .{ }^{1}$ Let $S(x)$ denote the computation of four S-Boxes and $L(x)$ the linear transformation which is expressed using the left circular shift (rotation) operator («). We may write the $f$ component as

$$
\begin{aligned}
S(x) & =S_{1}\left(x_{0}\right)\left|S_{2}\left(x_{1}\right)\right| S_{3}\left(x_{2}\right) \mid S_{4}\left(x_{3}\right) \\
L(x) & =x \oplus(x \lll 6) \oplus(x \lll 10) \\
f(x) & =L(S(x)) .
\end{aligned}
$$

We further define a 16-bit keyed permutation WD16 using $f$ as

$$
\mathrm{WD} 16(x, a, b, c, d)=f(f(f(f(x \oplus a) \oplus b) \oplus c) \oplus d) .
$$

The inverse of $f(x)$ and WD16 can be derived in straightforward fashion.

\subsection{Initialization}

The internal state of Hummingbird-2 is initialized with a four-round procedure using the 64-bit nonce $I V$. We first set

$$
R^{(0)}=\left(I V_{1}, I V_{2}, I V_{3}, I V_{4}, I V_{1}, I V_{2}, I V_{3}, I V_{4}\right)
$$

and then iterate for $i=0,1,2,3$ the following:

$$
\begin{aligned}
t_{1} & =\mathrm{WD} 16\left(R_{1}^{(i)} \boxplus\langle i\rangle, K_{1}, K_{2}, K_{3}, K_{4}\right) \\
t_{2} & =\mathrm{WD} 16\left(R_{2}^{(i)} \boxplus t_{1}, K_{5}, K_{6}, K_{7}, K_{8}\right) \\
t_{3} & =\mathrm{WD} 16\left(R_{3}^{(i)} \boxplus t_{2}, K_{1}, K_{2}, K_{3}, K_{4}\right) \\
t_{4} & =\mathrm{WD} 16\left(R_{4}^{(i)} \boxplus t_{3}, K_{5}, K_{6}, K_{7}, K_{8}\right) \\
R_{1}^{(i+1)} & =\left(R_{1}^{(i)} \boxplus t_{4}\right) \lll 3 \\
R_{2}^{(i+1)} & =\left(R_{2}^{(i)} \boxplus t_{1}\right) \ggg 1 \\
R_{3}^{(i+1)} & =\left(R_{3}^{(i)} \boxplus t_{2}\right) \lll 8 \\
R_{4}^{(i+1)} & =\left(R_{4}^{(i)} \boxplus t_{3}\right) \lll 1 \\
R_{5}^{(i+1)} & =R_{5}^{(i)} \oplus R_{1}^{(i+1)} \\
R_{6}^{(i+1)} & =R_{6}^{(i)} \oplus R_{2}^{(i+1)} \\
R_{7}^{(i+1)} & =R_{7}^{(i)} \oplus R_{3}^{(i+1)} \\
R_{8}^{(i+1)} & =R_{8}^{(i)} \oplus R_{4}^{(i+1)} .
\end{aligned}
$$

\footnotetext{
${ }^{1}$ Some early versions of Hummingbird-2 used a different set of S-Boxes from Serpent [1]. Hummingbird-2 was tweaked in May 2011 to use these S-Boxes.
} 
The initial state for encrypting the first plaintext word is $R^{(4)}$. Note that the two's complement numerical value of $i=0 \ldots 3$ is used in computation of $t_{1}$.

\subsection{Encryption}

Encryption of a single word of plaintext $P_{i}$ to ciphertext word $C_{i}$ requires four invocations of WD16. ${ }^{2}$

$$
\begin{aligned}
t_{1} & =\mathrm{WD} 16\left(R_{1}^{(i)} \boxplus P_{i}, K_{1}, K_{2}, K_{3}, K_{4}\right) \\
t_{2} & =\mathrm{WD} 16\left(R_{2}^{(i)} \boxplus t_{1}, K_{5} \oplus R_{5}^{(i)}, K_{6} \oplus R_{6}^{(i)}, K_{7} \oplus R_{7}^{(i)}, K_{8} \oplus R_{8}^{(i)}\right) \\
t_{3} & =\mathrm{WD16}\left(R_{3}^{(i)} \boxplus t_{2}, K_{1} \oplus R_{5}^{(i)}, K_{2} \oplus R_{6}^{(i)}, K_{3} \oplus R_{7}^{(i)}, K_{4} \oplus R_{8}^{(i)}\right) \\
C_{i} & =\mathrm{WD16}\left(R_{4}^{(i)} \boxplus t_{3}, K_{5}, K_{6}, K_{7}, K_{8}\right) \boxplus R_{1}^{(i)} .
\end{aligned}
$$

After each encrypted / decrypted word, we perform the following state update:

$$
\begin{aligned}
& R_{1}^{(i+1)}=R_{1}^{(i)} \boxplus t_{3} \\
& R_{2}^{(i+1)}=R_{2}^{(i)} \boxplus t_{1} \\
& R_{3}^{(i+1)}=R_{3}^{(i)} \boxplus t_{2} \\
& R_{4}^{(i+1)}=R_{4}^{(i)} \boxplus R_{1}^{(i)} \boxplus t_{3} \boxplus t_{1} \\
& R_{5}^{(i+1)}=R_{5}^{(i)} \oplus\left(R_{1}^{(i)} \boxplus t_{3}\right) \\
& R_{6}^{(i+1)}=R_{6}^{(i)} \oplus\left(R_{2}^{(i)} \boxplus t_{1}\right) \\
& R_{7}^{(i+1)}=R_{7}^{(i)} \oplus\left(R_{3}^{(i)} \boxplus t_{2}\right) \\
& R_{8}^{(i+1)}=R_{8}^{(i)} \oplus\left(R_{4}^{(i)} \boxplus R_{1}^{(i)} \boxplus t_{3} \boxplus t_{1}\right) .
\end{aligned}
$$

A shorthand of this is $C=E(P)$. The state variable $R$ is stepped by one iteration for each invocation of $E$. Note that the update function can be simplified since certain terms are re-used.

\subsection{Authenticating fixed-length unencrypted associated data}

Authenticated Encryption with Associated Data (AEAD) is a method of using Hummingbird that encrypts / decrypts a payload and also authenticates any associated data (AD) that travels alongside the ciphertext such as the nonce and a packet header. AD processing is optional in implementations.

AD processing occurs after the entire encrypted payload has processed. We simply compute $E\left(A_{i}\right)$ but transmit $A_{i}$ itself instead. Note that the size of the $\mathrm{AD}$ must be fixed (known by the recipient).

\footnotetext{
${ }^{2}$ Some early versions of this paper had a typographic error here as the final addition of $R_{1}^{(i)}$ was missing. Thanks to Jean-Philippe Aumasson for spotting this.
} 


\subsection{Stream cipher mode: A technique for encoding short fixed-length fields}

Sometimes it is desirable to communicate, without message expansion, datagrams which are less than 16 bits in size. We describe an encoding technique which enables this.

Let $x$ be the short message of $1 \leq n \leq 15$ bits. The ciphertext message is derived from the $n$ least significant bits of $x \oplus E(0)$. If message integrity is required, the state is further updated by $E(x)$. Decoding is straightforward.

Note that encrypting the two words 0 and $x$ has exactly the same effect on state and hence special care must be taken to ensure that both parties are in sync. If a protocol requires arbitrary-length authenticated messages, this technique can be used for padding, but the total message length (in bits) must be specified and verified in an unambiguous fixed-length AD field.

\subsection{Computing the Message Authentication Code}

To compute a message authentication tag $T$ of $n \leq 8$ words, we first finalize the message by first stepping the cipher three times without producing any output.

$$
\begin{aligned}
& E\left(I V_{1} \boxplus R_{1} \boxplus R_{3} \boxplus n\right) \\
& E\left(I V_{2} \boxplus R_{1} \boxplus R_{3}\right) \\
& E\left(I V_{3} \boxplus R_{1} \boxplus R_{3}\right) .
\end{aligned}
$$

Here $R_{1}$ and $R_{3}$ denotes the contents of those register words immediately before each invocation of $E$. We then construct the words of the authentication tag $T$ as follows:

$$
\begin{aligned}
& T_{1}=E\left(I V_{4} \boxplus R_{1} \boxplus R_{3}\right) \\
& T_{i}=E\left(R_{1} \boxplus R_{3}\right) \text { for } \mathrm{i}=2,3, \ldots, \mathrm{n} .
\end{aligned}
$$

\subsection{Uniqueness Requirement for IVs and Keys}

Hummingbird-2 is an authenticated encryption primitive and may be used in similar fashion as the Galois/Counter Mode (GCM) and GMAC, which are part of NSA "Suite B" algorithms. Implementers would be wise to take similar care in ensuring that keys and IVs are never repeated. We restate the requirement from Section 8 of [7].

The probability that the authenticated encryption function ever will be invoked with the same IV and the same key on two (or more) distinct sets of input data shall be no greater than $2^{-32}$. 
Generally speaking, compliance to this requirement is recommended in order to mitigate the risk of using Hummingbird-2 in certain applications, and this cipher is not as vulnerable to repeated IV attacks as AES-GCM and should be able to resist many realistic chosen-IV attacks.

Indeed Hummingbird-2 leaks very little information should the nonce be repeated. At most all you get are exact repetitions in the ciphertext where you have exact repetitions in the plaintext.

\section{Development and Analysis}

The main differences between Hummingbird-1 and Hummingbird-2 are:

- The key size has been set to 128 bits to be commensurable with the actual security of the cipher.

- The state size of the cipher has been increased from 80 bits to 128 bits and the LFSR has been eliminated. The last four new state registers $R_{5}, R_{6}, R_{7}$, and $R_{8}$ are called the "accumulator" registers.

- The keyed transform, called the "E Box" in [20] now only has four invocations of the S-Boxes, compared to five in Hummingbird-1. This increases the encryption speed of the cipher.

- The authentication mechanism has been improved to thwart a message extension attack.

- Support for authenticating unencrypted associated data has been introduced with the AEAD mode discussed in Section 2.4.

- Recommendations to the reuse of keys and IVs have been introduced.

- An important design criteria was compatibility with the ISO 18000-6C timing requirements as discussed in Section 5.

We note that prior to publication, Hummingbird-2 has been subjected to a significant cryptanalytic security assurance effort. For this, the services of Jim Frazer \& Son Cryptography (formerly ISSI) and the U. Waterloo Centre for Applied Cryptography Research were used, in addition to input from public analysis of Hummingbird-1. After thousands of hours of cryptanalysis, no significant flaws or sub-exhaustive attacks against Hummingbird-2 have been found. We summarize the results of our analysis in the following sections.

\subsection{Structure of the Cipher}

Hummingbird-2 has been designed to be as lightweight as possible while still maintaining a reasonable security margin against attacks. The state size of 128 bits should not be confused with the state size of stream ciphers (where the 
key is usually loaded into the evolving state registers upon initialization). In Hummingbird-2 the key is kept constant and hence we could say that the state is $128+128=256$ bits of which 128 bits is evolving. There is strong experimental and theoretical evidence that the cycle upon constant input words is $2^{127}$.

The initialization function is one-to-one from the IV to the four state registers $R_{1}, R_{2}, R_{3}$, and $R_{4}$. Hence there are no nonce collisions. If more than $2^{64}$ invocations of the $E$ function is performed under a single key, a birthday condition in the internal state may occur. The usage condition given in Section 2.7 will prevent this. Such a birthday condition has very limited cryptographic implication beyond serving as a distinguisher with that complexity. We note that this is the same bound that can be found for the AES algorithm [17].

\subsection{S-Box selection}

The S-Box (Table 1 and Section 2.1) selection was based on specific research presented in more detail in [21]. The S-Boxes also belong to the optimal classes discussed in [14].

We performed an exhaustive search through 16 ! possible permutations. The S-Boxes specifically belong to a classes that ideally satisfy the following conditions:

- Optimal differential bound $p \leq 1 / 4$, linear bound $\epsilon \leq 1 / 4$, and branch number 3.

- There is a minimum number of differential characteristics and linear approximations at the bounds.

- All S-boxes belong to a different linear equivalence class.

- The four S-boxes have a large Hamming distance from each other and the identity permutation.

- The algebraic degree of all but one output bit is 3 and each output bit is nonlinearly dependent on a maximum number of input bits.

\subsection{Differential cryptanalysis}

Hummingbird-2 has been designed to be resistant to Differential Cryptanalysis $[3,4]$. The most interesting findings in our research involve the high-bit differential $\Delta=8000$ which behaves in the same way under modular addition and XOR. Much of the nonlinearity for lower bits comes from interplay of these two operations. The four rotations in the initialization phase were introduced to increase the resistance of the cipher against certain related-key attacks.

We have verified that Hummingbird-2 is provably resistant against the types of attacks described by Saarinen in [20]. This was done by performing a search 
of all high-bit differentials in both initialization and actual encryption phases of the cipher.

Differentials in the encryption function Let $\mathrm{H}=8000$ denote the high bit differential and $x$ some undefined ciphertext differential. We found the following differentials that hold with probability 1 .

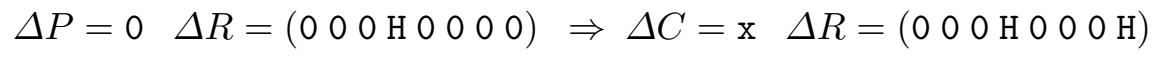

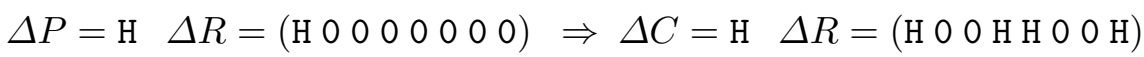

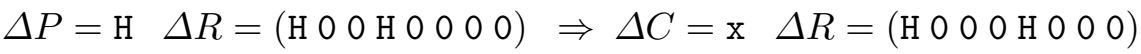

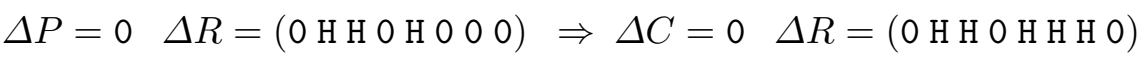

$$
\begin{aligned}
& \Delta P=\mathrm{H} \quad \Delta R=(\text { Н Н О Н О }) \Rightarrow \Delta C=\mathrm{H} \quad \Delta R=(\text { Н Н Н ОН Н }) \\
& \Delta P=\mathrm{H} \quad \Delta R=(\text { H H H O } 0) \Rightarrow \Delta C=\mathrm{x} \quad \Delta R=(\text { H Н ООН О) }
\end{aligned}
$$

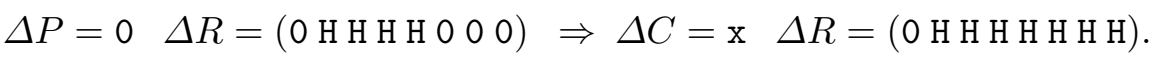

It can be observed that these differentials can't be used to construct an iterative differential.

Related keys in encryption For two related keys we found one iterative differential which holds with probability one. When the two keys are related by

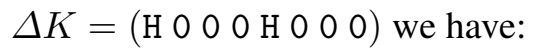

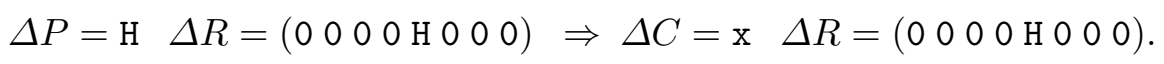

The ciphertext differential $\Delta C=x$ has some nontrivial value. We haven't found a direct way to exploit this related key property in an attack.

\subsection{Linear and Algebraic Cryptanalysis}

Hummingbird-2 has been designed to be resistant to Linear Cryptanalysis [15]. We performed a search for best linear masks in the mixing function $f$. Encryption of a single plaintext word involves sixteen invocations of $f$, five additions of state words ( $R_{1}, R_{2}, R_{3}, R_{4}$, and $R_{1}$ again) and XOR keying with both static keys and dynamic accumulator variables.

We ignore the modular additions in our analysis. The search and probability calculation was performed on up to four invocations of $f$, which is no longer distinguishable. Our findings give significant confidence to assert that Hummingbird-2 is resistant to linear cryptanalysis up to twelve rounds of $f$. We have also experimented with multiple linear approximations in our analysis [2]. 
The algebraic degree and branch number of the S-Boxes alone thwarts most forms of algebraic distinguishing attacks such as Cube Testers [6] and $d$-monomial distinguisers [19]. A typical black-box chosen-IV scenario is made difficult by the rather complicated initialization routine that has a total of sixteen WD16 invocations.

\section{Implementation and Performance}

Hummingbird-2 has been implemented in hardware and in software for various microcontroller architectures. Functions were written in assembly language and hand-optimized for all platforms. All library functions are C-callable and many development environments are supported.

\subsection{Microcontroller Software Implementations}

As Hummingbird-2 algorithm allows for trade-offs between implementation speed and size, we have implemented up to three software implementation profiles for some microcontroller platforms. Table 2 gives the characteristics of these implementations.

Table 2. Microcontroller software implementations of Hummingbird-2. Encryption and decryption speeds are given in cycles per 16-bit word.

\begin{tabular}{|l|l|l|l|l|l|l|}
\hline Target & Encr. & Decr. & Size & MAC-64 & Init. & RAM $\approx$ \\
\hline MSP430 “Tiny" & 1520 & 1544 & 770 & 10768 & 5984 & 50 \\
MSP430 "Fast" & 576 & 729 & 2518 & 4101 & 2187 & 114 \\
MSP430 "Furious" & 359 & 560 & 3648 & 2648 & 1361 & 114 \\
\hline AVR "Fast" & 745 & 930 & 3600 & 5689 & 2970 & 114 \\
AVR "Furious" & 574 & 770 & 4178 & 4310 & 2139 & 114 \\
AVR "Ultimate" & 495 & 652 & 3200 & 3764 & 1800 & 1500 \\
\hline PIC24 "Fast" & 319 & 371 & 2227 & 2248 & 1162 & 114 \\
PIC24 "Furious" & 271 & 362 & 4959 & 1897 & 912 & 114 \\
\hline ARM Cortex & 332 & 336 & 2200 & 2525 & 1492 & 116 \\
\hline
\end{tabular}

\subsection{Hardware Implementations}

Three hardware implementation profiles have been produced with differing size, power and speed characteristics. Table 3 summarizes these implementations. Figure 4.2 shows the layouts of the three cores. 
- High Performance Design HB2-ee4c. This 4 clock implementation of Hummingbird is targeted at fast encryption (4 clocks) and maximum throughput and bandwidth.

- Low Area and Power Design HB2-ee16c. This 16 clock implementation of Hummingbird is targeted at minimum area and power.

- Ultra Low Area and Power Design HB2-ee20c. This 20 clock implementation of Hummingbird is targeted at ultra low area and power.

The process used was TSMC $0.13 \mu \mathrm{m}$, operating with $1.2 \mathrm{~V}$. Encryption speed can be derived by dividing the operating frequency by the number of clocks required to encrypt a single word.

Table 3. Hardware implementations of Hummingbird-2. Encryption and decryption speeds are given in cycles per 16-bit word. The library used was TSMC 180nm, 6 level metal, high density. Synopsis synthesis tools were used.

\begin{tabular}{|l|l|l|l|l|l|l|}
\hline Profile & Frequency & $\begin{array}{l}\text { Clocks per } \\
\text { word }\end{array}$ & $\begin{array}{l}\text { Peak pwr } \\
(\mu \mathbf{W})\end{array}$ & $\begin{array}{l}\text { Leakage } \\
(\mu \mathbf{W})\end{array}$ & $\begin{array}{l}\text { Area } \\
\left(\mu \mathrm{m}^{2}\right)\end{array}$ & $\begin{array}{l}\text { Gate } \\
\text { Equiv. }\end{array}$ \\
\hline $\mathrm{HB} 2-\mathrm{ee} 4 \mathrm{c}$ & $100 \mathrm{kHz}$ & 4 & 1.93 & 4.17 & 27381 & 3220 \\
$\mathrm{HB} 2-\mathrm{ee} 4 \mathrm{c}$ & $10 \mathrm{MHz}$ & 4 & 163.1 & 4.17 & 27381 & 3220 \\
\hline $\mathrm{HB} 2-\mathrm{ee} 16 \mathrm{c}$ & $100 \mathrm{kHz}$ & 16 & 1.845 & 2.85 & 20871 & 2332 \\
$\mathrm{HB} 2-\mathrm{ee} 16 \mathrm{c}$ & $10 \mathrm{MHz}$ & 16 & 156.8 & 2.85 & 20871 & 2332 \\
\hline $\mathrm{HB} 2-\mathrm{ee} 20 \mathrm{c}$ & $100 \mathrm{kHz}$ & 20 & 1.73 & 2.63 & 19383 & 2159 \\
$\mathrm{HB} 2-\mathrm{ee} 20 \mathrm{c}$ & $10 \mathrm{MHz}$ & 20 & 149.1 & 2.63 & 19383 & 2159 \\
\hline
\end{tabular}
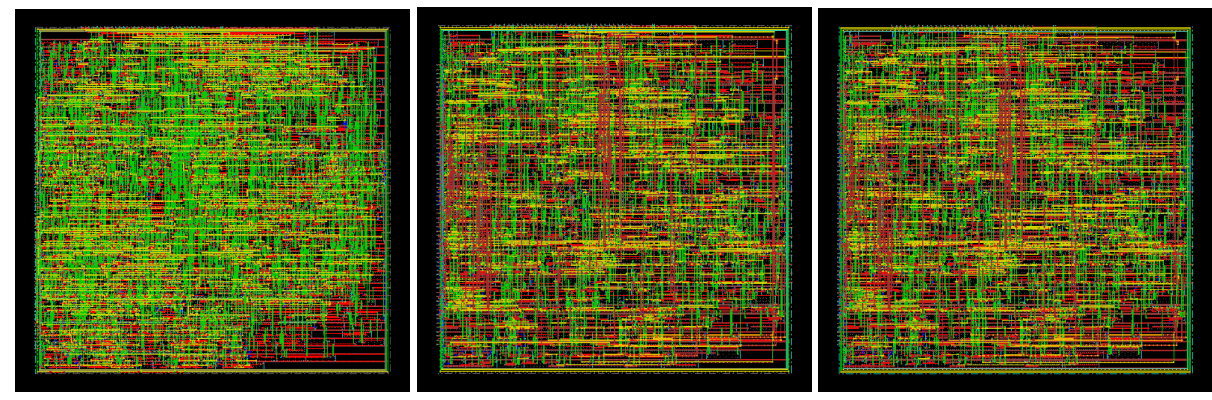

Fig. 1. From left: The layouts of HB2-ee4c, HB2-ee16c, and HB2-ee20c in $0.13 \mu \mathrm{m}$ process. 


\section{HB2 Timing Compatibility with ISO 18000-6C}

The ISO 18000-6C protocol [12] is the leading passive UHF RFID protocol in terms of number of tags sold today. The 18000-6C protocol specifies only a 32-bit password for access control and an electronic kill function.

The primary functionality of the $18000-6 \mathrm{C}$ protocol is for fast and efficient tag identification across a range of operating environments. Consequently, $18000-6 \mathrm{C}$ supports a range of data rates at which the interrogator and the tag may communicate. Communications are controlled by the interrogator in a Reader Talks First communication scheme. The interrogator begins a communication sequence by issuing a preamble that defines the length of the logic 0 and logic 1 symbols. The length of the logic 0 symbol is referred to as Tari, which is a fundamental timing parameter for communications.

For all commands except the Write command, the Tari value determines the amount of time the tag has to begin its response to the reader after the reader has completed the last symbol in its command to the tag. This time is referred to as T1 time. Table 4 shows the T1 timing for the minimum Tari value of 6.25 $\mu \mathrm{s}$, the maximum Tari value of $25 \mu \mathrm{s}$, and a commonly used Tari value of 12.5 $\mu \mathrm{s}$.

Table 4. T1 Timing Values and Available Clock Cycles.

\begin{tabular}{|c|c|c|c|c|}
\hline Tari $(\mu \mathbf{s})$ & T1 Time $(\mu \mathbf{s})$ & Cycles 1.5 MHz & Cycles 2 MHz & Cycles 2.5 MHz \\
\hline 6.25 & 39.06 & 58 & 78 & 97 \\
12.5 & 78.125 & 117 & 156 & 195 \\
25 & 187.5 & 281 & 375 & 468 \\
\hline
\end{tabular}

In addition to the $\mathrm{T} 1$ timings, Table 4 shows the number of full clock cycles available for computation within $\mathrm{T} 1$ for three on tag clock frequencies around 2 $\mathrm{MHz}$, a common on-tag clock frequency.

Table 5 compares the clocks per bit required to encrypt a single block for various ciphers that can be implemented with up to approximately three thousand gate equivalents and are therefore fit for RFID use. The figures for Katan have been derived from [5], for Present, ICEBERG and AES from [18] and for Trivium and Grain from [13].

Based upon the clocks per bit for each cipher, in Table 6 we compare the number of clocks required to encrypt various amounts of data.

Command decode and processing overhead may be considerable. Furthermore, the mode overhead and initialization of the basic block ciphers is not 
Table 5. Clocks Per Bit.

\begin{tabular}{|l|c|c|c|}
\hline Cipher & Block Size (bits) & Key Size (bits) & Clocks Per Bit \\
\hline HB2 & 16 & 128 & 0.25 \\
Grain-128 & 1 & 128 & 1 \\
Trivium & 1 & 128 & 1 \\
Present-80 & 64 & 80 & 0.5 \\
Present-128 & 64 & 128 & 0.5 \\
Katan32 & 32 & 80 & 8 \\
Katan48 & 48 & 80 & 5.31 \\
Katan64 & 64 & 80 & 3.98 \\
Iceberg & 64 & 128 & 0.25 \\
AES-128 & 128 & 128 & 1.25 \\
\hline
\end{tabular}

Table 6. Comparison of Clock Cycles to Encrypt. Note that most block ciphers require initialization every time key is changed.

\begin{tabular}{|l|c|c|c|c|c|c|c|}
\hline Cipher & Init & 16 bits & 32 bits & 48 bits & 64 bits & 96 bits & 128 bits \\
\hline HB2 & 16 & 4 & 8 & 12 & 16 & 24 & 32 \\
Grain-128 & 513 & 16 & 32 & 48 & 68 & 96 & 128 \\
Trivium & 1333 & 16 & 32 & 48 & 68 & 96 & 128 \\
Present-80 & $0^{*}$ & 32 & 32 & 32 & 32 & 64 & 64 \\
Present-128 & $0^{*}$ & 32 & 32 & 32 & 32 & 64 & 64 \\
Katan32 & $0^{*}$ & 256 & 256 & 512 & 512 & 768 & 1024 \\
Katan48 & $0^{*}$ & 255 & 255 & 255 & 510 & 510 & 765 \\
Katan64 & $0^{*}$ & 255 & 255 & 255 & 255 & 510 & 510 \\
Iceberg & $0^{*}$ & 16 & 16 & 16 & 16 & 32 & 32 \\
AES-128 & $0^{*}$ & 160 & 160 & 160 & 160 & 160 & 160 \\
\hline
\end{tabular}

considered. This additional processing will require even more overhead. While some operations may be performed in parallel, the command decode and processing must be performed prior to any cryptographic functions being performed.

In conclusion, HB2 is well suited for use in passive RFID systems due to its low power consumption, which minimally impacts range, and its high speed that enables the tag to continue normal operation within T1 timings.

\section{Conclusions}

We have presented Hummingbird-2, a lightweight authenticated encryption algorithm that we believe to be resistant to all standard attacks to block ciphers and stream ciphers such as differential and linear cryptanalysis, structure attacks and various algebraic attacks. Hummingbird-2 also has the further advantage of being resistant to chosen-IV attacks. 
We have also presented results of software and hardware implementations of Hummingbird-2. Hummingbird-2 can be implemented with little more than 2000 gate equivalents, making it well suited for ubiquitous devices such as RFID tags and sensors. Hummingbird- 2 has the additional advantage over other lightweight encryption primitives that it produces a message authentication code.

Acknowledgements. In addition to the anonymous RFIDSec ' 11 program committee members, we would like to thank Whitfield Diffie (who designed the original WD16 function) and the members of CACR and ISSI teams. Troy Hicks and Ken Lauffenburger were behind the hardware work on Hummingbird-2. Jared Smothermon, Stanford Hudson, and Bob Nimon did the Software implementations on various platforms.

\section{References}

1. R. Anderson, E. Biham And L. Knudsen. "Serpent: A Proposal for the Advanced Encryption Standard." http://www.cl.cam.ac.uk/ rja14/Papers/serpent. pdf (1999)

2. A. Biryukov, C. De Cannière And M. Quisquater. "On Multiple Linear Approximations." CRYPTO 2004, LNCS 3152, pp. 1-22. Springer (2004)

3. E. Biham And A. ShamiR. "Differential Cryptanalysis of DES-like cryptosystems." In A. Menezes and S.A. Vanstone (Eds.): CRYPTO 1990. LNCS 537, pp. 2-21. Springer (1990)

4. E. BihAm AND A. SHAmiR. "Differential Cryptanalysis of the Data Encryption Standard." Springer (1993)

5. C. De Cannière, O. Dunkelman and M. KneŽević. "KATAN \& KTANTAN - A Family of Small and Efficient Hardware-Oriented Block Ciphers." CHES 2009, LNCS 5747, pp. 272-288. Springer (2009)

6. I. Dinur And A. Shamir. "Cube Attacks on Tweakable Black Box Polynomials." EUROCRYPT 2009, LNCS 5479, pp. 278-299. Springer (2009)

7. M. DwORKIN. "Recommendation for Block Cipher Modes of Operation: Galois/Counter Mode (GCM) and GMAC." NIST Special Publication 800-38D (2007)

8. X. Fan, H. Hu, G. Gong, E. M. Smith and D. Engels. "Lightweight Implementation of Hummingbird Cryptographic Algorithm on 4-Bit Microcontroller." The 1st International Workshop on RFID Security and Cryptography 2009 (RISC'09), pp. 838-84. Springer (2009)

9. N. Ferguson, D. Whiting, B. Schneier, J. Kelsey, S. LuCKs, And T. Kohno "Helix: Fast Encryption and Authentication in a Single Cryptographic Primitive." FSE 2003, LNCS 2887, pp. 330-346. Springer (2003)

10. D. Engels, X. Fan, G. Gong, H. Hu And E. M. Smith. "Ultra-Lightweight Cryptography for Low-Cost RFID Tags: Hummingbird Algorithm and Protocol." Centre for Applied Cryptographic Research (CACR) Technical Reports, CACR-2009-29. http: / / www . cacr.math. uwaterloo.ca/techreports/2009/cacr2009-29.pdf (2009)

11. D. Engels, X. Fan, G. Gong, H. Hu and E. M. Smith. "Hummingbird: UltraLightweight Cryptography for Resource-Constrained Devices." 1st International Workshop on Lightweight Cryptography for Resource-Constrained Devices (WLC'2010). Tenerife, Canary Islands, Spain, January. (2010) 
12. INTERNATIONAL STANDARDIZATION ORGANIZATION. "ISO/IEC 18000-6:2010. Information technology - Radio frequency identification for item management - Part 6: Parameters for air interface communications at $860 \mathrm{MHz}$ to $960 \mathrm{MHz} . "$

13. T. GoOd AND M. BENAISSA. "Hardware results for selected stream cipher candidates." eStream, ECRYPT Stream Cipher Project Report 2007 / 023. Proceedings of SASC 2007 (2007)

14. G. Leander and A. Poschmann. "On the Classification of 4 Bit S-Boxes." In C. Carlet and B. Sunar (Eds.): WAIFI 2007, LNCS 4547, pp. 159-176. Springer (2007)

15. M. MATSUI. "Linear cryptoanalysis method for DES cipher.” In T. Helleseth (Ed.): EUROCRYPT 1993. LNCS 765, pp. 386-397. Springer (1993)

16. F. Muller. "Differential Attacks against the Helix Stream Cipher." FSE 2004, LNCS 3017, pp. 94-108. Springer (2004)

17. National Institute of Standards and Technology. "The Advanced Encryption Standard (AES).” FIPS Publication 197, U.S. DoC/NIST (2001)

18. A. PoschmanN "Lightweight Cryptography - Cryptographic Engineering for a Pervasive World". PhD Thesis. Europaeischer Universitaetsverlag, in the IT-Security series, no 8. ISBN 978-3899663419. Also available as IACR ePrint 2009/516 (2009)

19. M.-J. O. SAARINEN. "d-Monomial Tests are Effective Against Stream Ciphers." State of the Art in Stream Ciphers (SASC) 2006. Workshop Record, K.U. Leuven (2006)

20. M.-J. O. SAARINEN. "Cryptanalysis of Hummingbird-1.” FSE 2011, LNCS 6733, 328341. Springer (2011)

21. M.-J. O. SAARINEn. "Cryptographic Analysis of All $4 \times 4$ - Bit S-Boxes." Selected Areas in Cryptography (SAC) 2011. 11-12 August 2011, Toronto, Ontario, Canada, (2011)

22. D. Whiting, B. Schneier, S. Lucks, And F. Muller. "Phelix - Fast Encryption and Authentication in a Single Cryptographic Primitive.” ECRYPT Stream Cipher Project Report 2005/027. http://www.schneier.com/paper-phelix.html, 2005.

\section{A Test Vectors}

The test data is given as an array of bytes. When using these test vectors, note that Hummingbird-2 processes data in little-endian fashion (this means that the first 16-bit plaintext word in the second test vector is actually $0 \times 1100$ ).

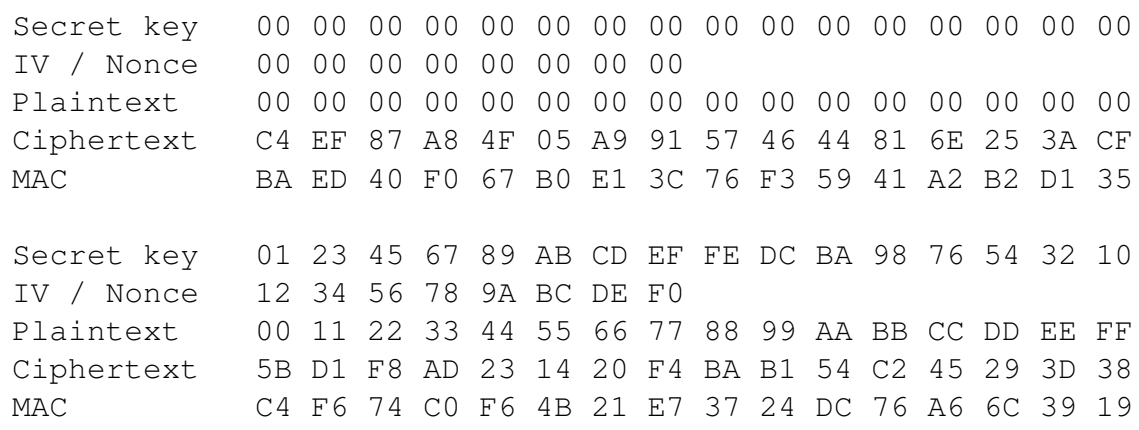

Document version 20110712121300. 